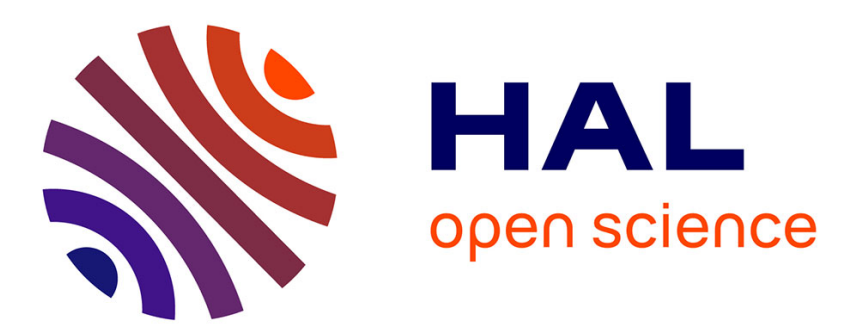

\title{
Co-Visualization of Air Temperature and Urban Data for Visual Exploration
}

\author{
Jacques Gautier, Mathieu Brédif, Sidonie Christophe
}

\section{To cite this version:}

Jacques Gautier, Mathieu Brédif, Sidonie Christophe. Co-Visualization of Air Temperature and Urban Data for Visual Exploration. IEEE VIS 2020, Oct 2020, Virtual Conference, United States. hal02985484

\section{HAL Id: hal-02985484 \\ https://hal.science/hal-02985484}

Submitted on 2 Nov 2020

HAL is a multi-disciplinary open access archive for the deposit and dissemination of scientific research documents, whether they are published or not. The documents may come from teaching and research institutions in France or abroad, or from public or private research centers.
L'archive ouverte pluridisciplinaire HAL, est destinée au dépôt et à la diffusion de documents scientifiques de niveau recherche, publiés ou non, émanant des établissements d'enseignement et de recherche français ou étrangers, des laboratoires publics ou privés. 


\title{
Co-Visualization of Air Temperature and Urban Data for Visual Exploration
}

\author{
Jacques Gautier* $\quad$ Mathieu Brédif ${ }^{\dagger} \quad$ Sidonie Christophe \\ LASTIG, Univ Gustave Eiffel, ENSG, IGN, F-94160 Saint-Mande, France
}

\begin{abstract}
Urban climate data remain complex to analyze regarding their spatial distribution. The co-visualization of simulated air temperature into urban models could help experts to analyze horizontal and vertical spatial distributions. We design a co-visualization framework enabling simulated air temperature data exploration, based on the graphic representation of three types of geometric proxies, and their co-visualization with a 3D urban model with various possible rendering styles. Through this framework, we aim at allowing meteorological researchers to visually analyze and interpret the relationships between simulated air temperature data and urban morphology.
\end{abstract}

Index Terms: Human-centered computing-VisualizationGeographic visualization;-_-Visualization design and evaluation methods; Visualization techniques-

\section{INTRODUCTION}

Understanding urban climate phenomena is one of the main issues regarding global warming. Amongst others, the urban heat island effect (UHI; [18]) causes higher temperatures in cities than in rural areas, accentuated during heat waves episodes and characterized by the lack of night-time cooling. UHIs are largely addressed [2, 7, 19,22], regarding diverse causes such as ground artificialization in cities, lower albedo causing more solar energy absorption, building density and higher urban surface roughness causing reduced air velocity $[3,5,15,17,21,24]$. A better understanding of the urban heat islands needs then more accurate knowledge of urban morphology [21,27]. Climate models simulate temperature, wind or humidity distributions and values, based on multiple indicators, including vegetation ratio and mean albedo of buildings surface, such as the Local Climate Zone (LCZ) classification characterizing regions by surface cover, structure, material and human activity [21, 23, 28]. Co-visualizing simulated climate data with morphological urban data would allow supporting the validation of climate simulation models, by comparing simulation results with morphological indicators used as simulation input data, such as a suitable visual analysis of relationships between UHIs and the urban environment. Regarding specifically temperature data, existing visualizations work as final media, to present global trends, breaks and changes, based on long-time series of temperature values as impactful stripes [8] or as spatialized data $[12,25]$, or to inform and explain the temperature profiles as vertical and horizontal cross-sections at city scale. The visualization of plane representations of air temperature into the city have been proposed, projecting temperature into 3D urban space [20], or representing local effects of urban structures by representing temperature on static/animated vertical cross-sections [10].

Meteorology researchers claim for more local and finer visual exploration of their simulated data integrated into real urban data, to ease the interpretation of relationships between urban heat islands and urban morphology. They particularly expect to analyze together

\footnotetext{
*e-mail: jacques.gautier@ign.fr

†e-mail:mathieu.bredif@ign.fr

‡e-mail:sidonie.christophe@ign.fr
}

or separately horizontal and vertical spatial distributions of air temperature data: horizontally, at the building block level, which is at the time the finest possible spatial resolution for atmospheric simulation models (air temperature data is now typically simulated with $500 \mathrm{~m}-2 \mathrm{~km}$ resolutions); vertically to observe vertical profiles into and above the city. Co-visualizing climate and urban morphological data, at the building block level, is less explored, because it requires precise urban representation data and models.

We present an integrated framework for co-visualizing urban simulated air temperature and urban morphology, at a building block level, which remains complex because of the following data characteristics and their possible visual integration into a 3D scene:

- 3D Urban model. A topographic model of metric resolution enables the visualization of buildings, blocks, shapes, heights, structures, empty area, urban canyon depth and direction.

- Temperature data, simulated by atmospheric models, is sampled on a 3D grid (Fig.1.left) which is :

- Horizontally: a regular square grid. Horizontal resolution being larger than the resolution of the urban model, this scale difference may cause over-interpretation.

- Vertically: grid sample altitudes start at the ground level, following the topographic elevation, with a spacing that gradually goes from metric at the ground level to much sparser in the levels above the urban canopy.

- Co-visualization of simulated air temperature and urban morphology implies to visually integrate, into a 3D visualization environment, heterogeneous and multi-dimensional data, with various spatial resolutions, geometries and data structures.

These characteristics of the data, and so the characteristics of their co-visualization, require to work on the data possible graphic representation and rendering styles, in order to ease their visual integration and representation (lowering visual heterogeneity, avoiding visual clutter), and on the interaction capacities which would support the navigation into the 3D scene to explore the data and the visual analysis of their spatial relationships. In order to fit these expectations, we design and develop a co-visualization framework based on the representation of air temperature data as geometric proxies, integrating and transforming the initial irregular 3D grid into horizontal planes, 3D point clouds, or road-based vertical planes, and their related style capacities and interaction modes. We aim at providing tools, to help the users to navigate into those graphic representations of air temperature data, and to validate and/or to open questions about UHI explanations and interpretations.

\section{Related work}

Visualization aims at supporting visual perception and interpretation of data, enhanced by data interaction to go to visual analytics. In the scope of geovisualization, we handle issues regarding geospatial or spatialized data visualization. Especially we target spatio-temporal phenomena on Earth, that could be represented by raw, predicted, simulated, or learned data coming from various acquisition sensors and input models, and their related imprecision, to be (visually) propagated. In particular, climate data visualization attempts mostly to verify input/output data and models, and demonstrate global and 


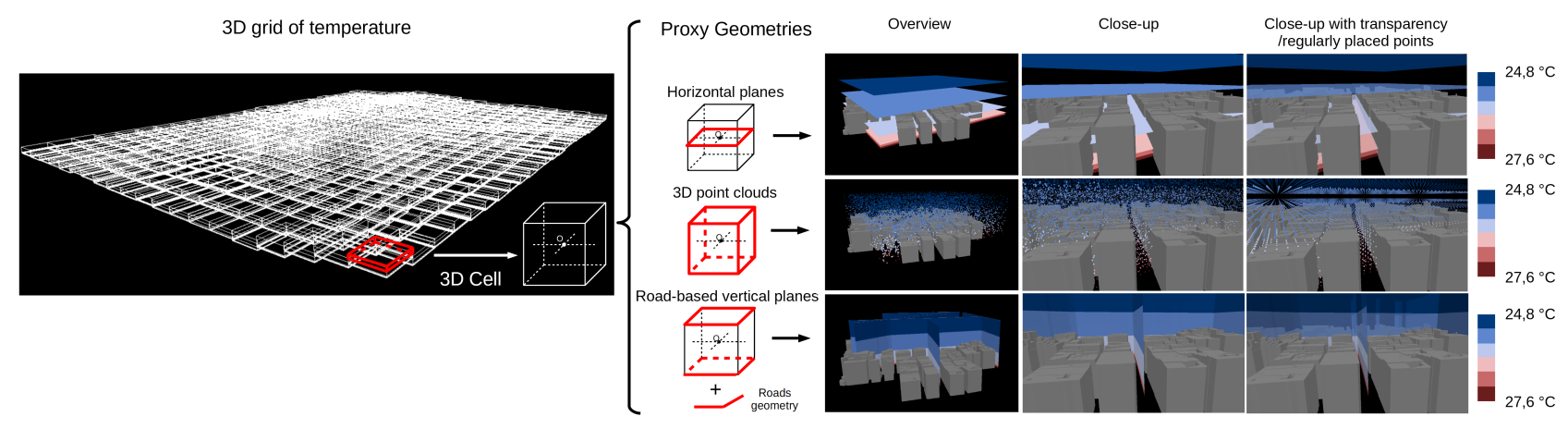

Figure 1: From the 3D grid of temperature data (left) to geometric proxies (middle), stylized and co-visualized with urban data (right).

local patterns, whereas urban climate data visualization focuses more on spatialization of climate data, and visual exploration of spatial relationships between climate specificities and urban morphology.

\subsection{Climate data Visualization}

Climate data visualization mostly exists to validate and communicate about simulation results, and especially about air temperature profiles, dynamic wind representation [13] or possible air corridors [4], with the expectations to validate input/output data and models characteristics. Many representation types are used, most of all as vertical and horizontal cross-sections, long time series spatialized data [12] or global trends into long time series [8]. Sets of multi-dimensional spatial 2D views from very small scales (at European level for instance), to wider scales (at city scales for instance) are very often proposed to visualize the spatial distribution of air temperature coming from simulation models integrating morphological indicators, in addition to visualizations of their temporal profile distributions through graphs and histograms $[12,25]$. Spatio-temporal animations are very useful to improve the global perception of a spatio-temporal phenomenon and its general patterns, but it remains difficult to detect and analyze changes, at any scales. In addition, the beautiful and so efficient graphic representation of warming stripes for climate data visualization is particularly relevant and has a strong public impact [8]. These graphic representations are very powerful to inform and drive visual attention on global trends. However, they have limitations to support advanced visual analytics, and more local approaches, even spatial or temporal: actually they do not offer other interaction capacities to manipulate directly the data and explore them, in order to infer some other types of knowledge.

\subsection{Urban climate Visualization}

Visualizing climate data in their urban environment is a way both to validate simulation models and to analyze the relationships between input indicators and data of the models and simulated data with urban morphology. Plane representations of aggregated ground or air temperature, are mainly displayed at city scales, due to the limit of spatial resolution. Those spatialized representations are displayed as planes into a $3 \mathrm{D}$ environment or projected on $3 \mathrm{D}$ volumes of buildings, in order to have another point of view on the spatial resolution of the represented information. Playing with possible dimensions of the 3D environment allows using ground and building visual variables to represent $2 \mathrm{D}$ and $3 \mathrm{D}$ scalar data: for instance, the air quality data represented by the color of the ground [10], the representation of the simulated temperature of buildings surface [29], or using colored buildings to represent simulated air temperature near the ground surface [20]. Existing scientific visualization libraries and softwares such as Paraview [1], ANSYS Discovery Live [9] and VTKm [16] propose different renderings of multi-dimensional data such as climate data, based on the representation of iso-volumes or $3 \mathrm{D}$ slices of 3D data. Such representations are interesting to explore respectively the spatial distribution of specific temperature interval, or the value of temperature data at a specific location. These tools are efficient to represent the physics of a phenomenon, but avoid to go deeper in its spatial analysis. Color palettes for geophysics phenomena are still in question to improve scientific analysis and sharing $[6,26]$, but also remain questionable. Those considerations reach to graphic semiology issues to help visual perception and spatial cognition, based on suitable and efficient visualizations.

Due to both climate and urban data dimensions, we propose to design and implement the representation of air temperature as particular 3D data: enabling graphic representation and interaction would provide a co-visualization framework to represent and explore temperature data in their urban spatial context at finer spatial scale.

\section{3D URBAN TEMPERATURE EXPLORATION}

The air temperature data co-visualized in this work was simulated by the two following atmospheric models: Meso-NH model (Mesoscale Non-Hydrostatic atmospheric model), simulating climate data for the atmosphere until altitudes of 15000 meters [11]; TEB model (Town Energy Balance), extending the Meso-NH simulations in the urban environment, below the building roofs [14]. Simulated air temperature is represented by a 3D grid (Fig.1.left). Each value is related to the center of one 3D cell (Point O) of the grid (Fig 1.middle). We consider the temperature inside each cell as homogeneous and equal to the temperature at the cell's center, in order to not introduce understanding bias about the simulated temperature values, by directly visualizing the outputs of the simulation models.

On this basis, we follow these design rules: see the overall complexity at city scale, understand temperature value evolution according to altitude and XY dimensions, avoiding occlusion effects with buildings and with the geometric representation of temperature data, enabling city exploration at any relevant scale and camera position, supporting the analysis of altitude effects on temperature, or the relationship between urban morphology and temperature.

\subsection{Geometric proxies for temperature representation}

Three geometric proxies are specified to represent the main characteristics of simulated air temperature represented as an irregular 3D grid, i.e. its irregular horizontal and vertical distributions: horizontal planes, 3D point clouds, road-based vertical planes. Providing different geometric proxies will help users to explore data with different points of view, enabling a better co-visibility of data. Each proxy is described by its geometric structure and its style capacities. Temperature values are represented by a discrete color scale, representing equal intervals of temperature values from the highest, near the ground (red) to the lowest above the buildings (blue).

\subsubsection{Horizontal planes}

In order to analyze and compare horizontal distributions at different height levels above the ground, temperature data are represented 
by stacks of horizontal planes. Each stack represents a vertical column of 3D cells: the altitude of each plane corresponds to the altitude of the center of the corresponding cell, e.g. is represented at equal distance from the top and bottom of the cell (Fig.1.middle). Horizontal planes at the same height level are nearly coplanar (with offsets due to ground elevation variations) and represent the XYdistribution of temperature, at $650 \mathrm{~m}$ of resolution. Rendering stacks of horizontal planes brings an issue of occlusion between planes, according to the camera position, the visualization scale and the urban context of buildings: transparency may help to make visible different temperatures into several horizontal planes.

\subsubsection{D point clouds}

Our expectations for this proxy geometry is to make more visible the horizontal and vertical distributions of temperature into a potential cluttered visual environment, because of accumulated/combined occlusions of buildings and other levels of temperature, as well as improving the visualization of the $3 \mathrm{D}$ cell borders. Temperature data are represented by a point cloud filling the volume corresponding to the 3D cell (Fig.1.middle). The whole data is thus represented by stacks of 3D point clouds, whose density and point size can be calibrated by the user. Different ways are explored to construct the point cloud, by regularly or randomly positioning the points.

\subsubsection{Road-based vertical planes}

To improve the visualization of the temperature vertical distribution, the values are displayed along the vertical axis on a continuous geometry, without the discontinuity of the point clouds and the horizontal planes. Temperature data are represented by vertical planes, of equivalent height of the $3 \mathrm{D}$ cells. One stack of vertical planes represents the temperature vertical distribution at some XY-location, each plane representing a slice of temperature (Fig.1.middle). The planes are located in the middle of urban roads, and rendered by extruding vertically the linear geometry of the road network. In order to answer possible occlusion issues, transparency can be applied to the vertical planes.

\subsection{Visual analysis of temperature spatial distribution}
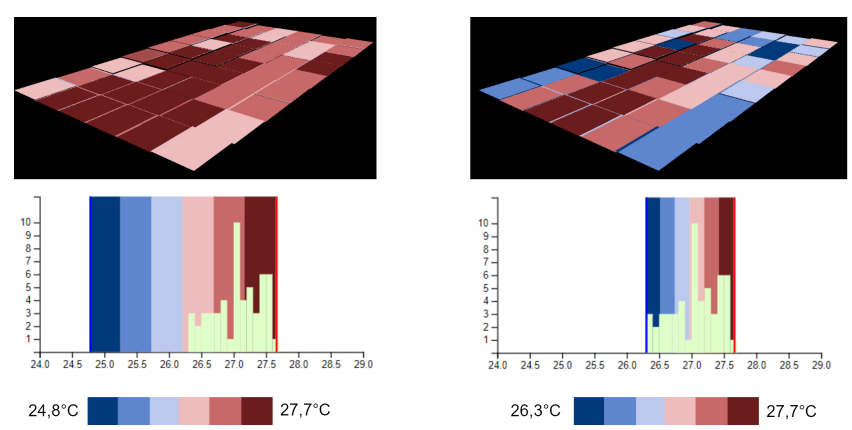

Figure 2: Interactive histogram of temperature value distribution (green), to edit the temperature range (discrete color scale).

As a first part of a typical use case, temperature data exploration enables the visual analysis of their horizontal and vertical distributions, while highlighting extreme values and spatial gradients at different scales. We design an interactive histogram showing the visualized temperature values distribution, in which a discrete color scale can be associated with a value range. According to the set range, the value intervals related to colors are represented in the histogram's background. After changing the range boundaries, the new color scale is applied in the 3D scene (Fig.2). To highlight spatial gradients with the $3 \mathrm{D}$ point cloud, we propose specific rendering methods. First, high/low values can be represented with a higher/lower density of points, and/or with a bigger/smaller point size, enhancing the spatial gradients (Fig.3). Secondly, data can be filtered according to their values, making visually salient some characteristics of the data. Finally, the filtering of the data values can be animated, displaying different value intervals in time, in order to identify potential spatial directions. Such (animated) filtering can be done according to the spatial position of points.

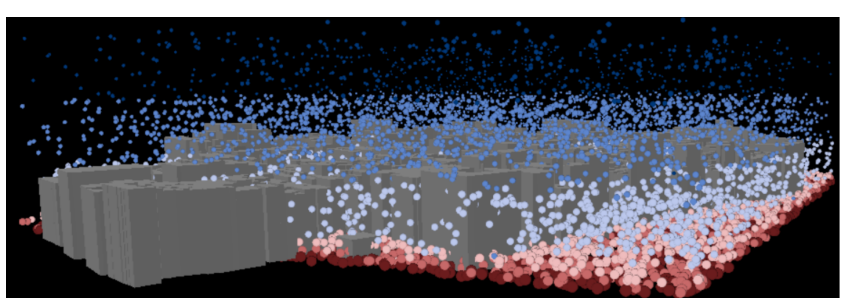

Figure 3: Enhancing spatial gradients using point style.

\subsection{Co-visualizing temperature and urban data}

As a second part of a typical use case, Temperature data can be co-visualized with the 3D urban model, which offers spatial contextualization. The co-visualization framework offers to jointly manage the style edition of the urban and temperature models. Each type of temperature representation can be stylized in different ways, according to the user's expectations regarding to the task, data, and scale. Buildings can be represented with opaque or transparent facades and roofs, or only by their footprints: for instance with uniform colors, or with LCZ classification colors (Cf. 3 columns respectively on the left, and on the right, in Fig. 4), in order to analyze spatial relationships between the temperature distribution and the urban morphology. Handling both styles facilitates the visual tracking and comparison, and allows avoiding occlusions.

\section{IMPLEMENTATION}

The framework is implemented as an open source Web application, available on GitHub, with a JQuery-based user interface. Temperature data are stored in NetCDF files, providing for each 3D cell its center's geographic coordinates and altitude. As a preprocess, these coordinates are projected in Lambert-93 spatial reference system. Buildings and roads data are stored in GeoJSON files, providing for each feature their vertex coordinates in Lambert-93 (and the building's height). Proxy and building geometries are processed from these data with Javascript with a vertical exaggeration of $3: 1$, and rendered using the Three.js WebGL library, with custom GLSL vertex and fragment shaders. Temperature values are uploaded as a 3D texture shared across all shaders, which has the same dimensions as the 3D grid (x:26, y:22, z:37). Using the 3D grid spatial characteristics (coordinates, altitudes), proxy geometry's coordinates are converted by the shaders to interrogate the $3 \mathrm{D}$ texture.

\section{Results and Discussion}

\subsection{Visual analysis of air temperature into city blocks}

We provide a suitable framework to visually integrate and interact with urban and air temperature data: three geometric proxies could be used alternatively by users, to display air temperature in the city. The continuous representation provided by the planes is expected to be effective to analyze the horizontal and vertical distribution of temperature values; the representation by $3 \mathrm{D}$ point clouds is expected to provide an analysis of temperature distribution in both horizontal and vertical dimensions, by seeing different levels of data through empty spaces between particles. Different refinements could be done to improve temperature distributions analysis, by modifying the color scale with the interactive histogram, or by handling filtering 


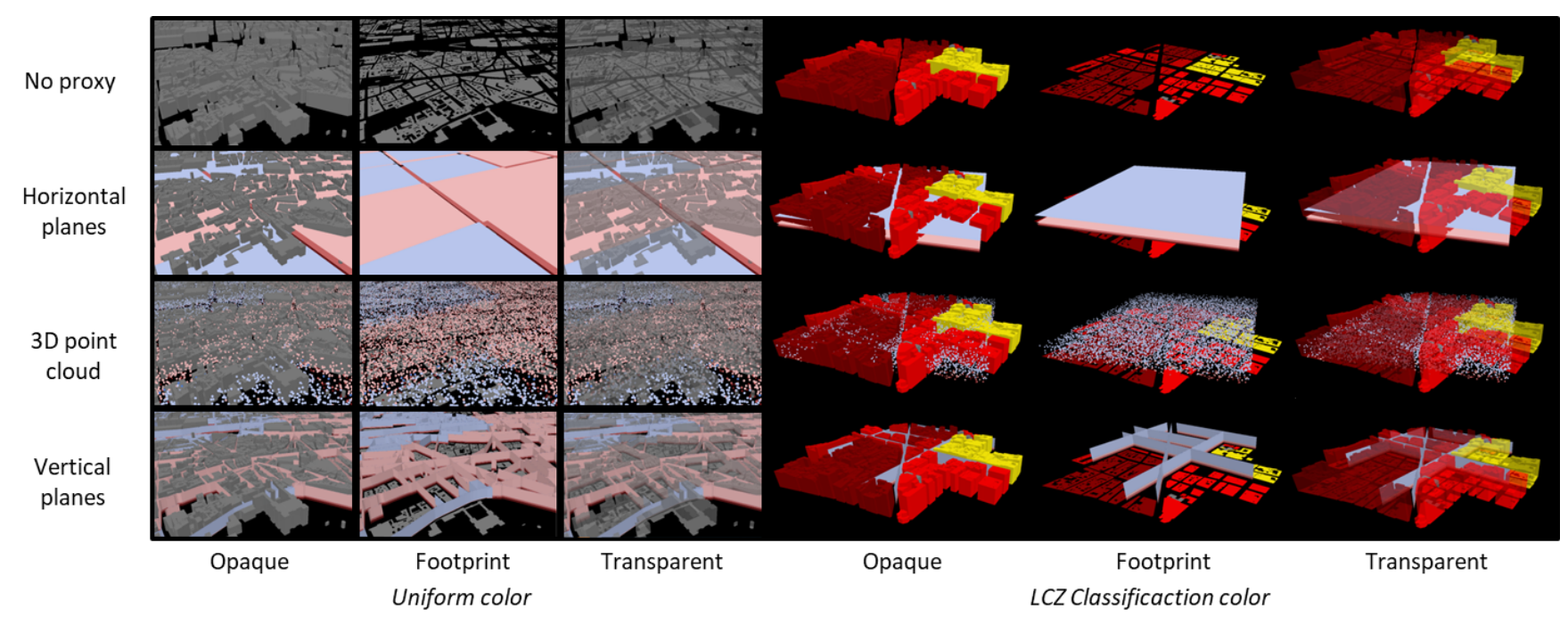

Figure 4: Co-visualization of urban data and air temperature data. On lines: Geometric proxies for temperature: none, horizontal planes, 3D point cloud, vertical planes. On columns: styles for buildings: in 2 modes, uniform or LCZ classification colors, opaque, footprints, transparent.

and animation methods for the point clouds. According to their ongoing task of data observation or analysis, the users could control the graphic representation of the city, while editing the buildings style for the whole scene. At any moment, the user could modify the camera view point and the required scale of visualization.

\subsection{Expert evaluation}

Two meteorological experimented researchers have been asked to evaluate the tool as end-users while co-visualising and interacting with temperature data and testing functionalities. Open discussions were held on the different temperature and building representations and their capacities to support the interpretation of the spatial distributions of air temperature data. We asked our end-users if they easily could handle the different representations and interactions, what was useful or not. Their expectations about potential new visualizations or interactions were also discussed. Their feedbacks confirm their interest and the efficiency of co-visualizing temperature data with a 3D city model at city block level: first, to validate their own simulated data, and second to analyze spatial relationships between temperature and urban data. They appreciate having the possibility to manipulate different ways to represent the same temperature data, helping them to refine their expectations, and to effectively support their exploration with various 'points of view' on the data. Having a controllable 3D urban model really matters to support urban navigation and recognition of the city, such as the identification of fresh areas (rivers, wooded area). Among the different proposed temperature representations, the end-users show clearly a preference for the vertical planes, to explore the horizontal and vertical distributions of the data. They especially appreciate the possibility to visualize how the horizontal distribution of temperature evolves with altitude. Horizontal planes are appreciated for the analysis of the horizontal distribution of temperature at specific levels, but transparency is not considered as efficient to visualize several vertical levels of data. Point clouds are controversial about the ease of the visual analysis of vertical profiles: when the purpose is to lower vertical occlusions, different values of temperature are effectively better identified through gaps between points, but the vertical order of these values seems difficult to perceive. They privilege regularly placed 3D points clouds to ease the perception of this vertical order Filtering or animating the 3D point cloud do not appear as effective as the continuous representation offered by the planes to identify a spatial gradient. The exploration of the distribution of specific ranges of temperature, through the modification of the color scale with the temperature histogram, is very appreciated.

\section{Conclusion AND Future Works}

Providing such a co-visualization framework of urban and air temperature data is a first step towards multidimensional exploration of urban climate data, in order to improve visual reasoning and interpretation on urban heat islands. For climate analysis purposes, visually integrating other data on building (facade temperature, energy losses, material quality, etc.), at city levels (ground temperature, land use cover, morphological and socio-demographic indicators, etc.), and other climate data (wind, rain, etc.) is at stake to infer new knowledge. A multidimensional visualization requires to possibly handle several simulations, with different resolutions and different related time steps, allowing their comparison along any possible dimension. Regarding geovisualization issues, adding new data will increase the visual and cognitive complexities of co-visualizing heterogeneous data, varying in space, time, altitude: this would require to design and combine new geometric structures and to extend style specifications in the co-visualization framework. Virtual reality could present an opportunity for the analysis of high-dimensional datasets. Temperature data exploration has also to be further investigated, while providing more tools to support the identification of daily variability: for instance, of 0,5 degrees at some point, and until 3 degrees by night during heatwaves episodes.

Because of the wide possible exploration space, the user exploration has to be driven by data resolution, scale, even data preanalysis, with the help of more advanced interaction capacities. By exploring different visualization methods for the visual analysis of 3D complex multi-scale and multi-dimensional simulated data about urban climate phenomena, this paper is part of a long-term research approach to bring simulation and visualization more closer. The on-going production of more accurate simulated data, at $250 \mathrm{~m}$ or even $150 \mathrm{~m}$ resolution, will allow going deeper into a subtle visual analysis of climate and urban interaction.

\section{ACKNOWLEDGEMENTS.}

This research work is granted by the URCLIM project as part of ERA4CS, an ERA-NET initiated by JPI Climate with co-funding of the European Union (Grant n690462). The climate data has been provided by V. Masson, leader of the project. We thank V. Masson and $\mathrm{M}$. Goret, for having accepted to evaluate our work. 


\section{REFERENCES}

[1] J. Ahrens, B. Geveci, and C. Law. Paraview: An end-user tool for large data visualization. The visualization handbook, 717, 2005.

[2] H. Akbari and D. Kolokotsa. Three decades of urban heat islands and mitigation technologies research. Energy and Buildings, 133:834-842, 2016.

[3] H. Akbari, M. Pomerantz, and H. Taha. Cool surfaces and shade trees to reduce energy use and improve air quality in urban areas. Solar energy, 70(3):295-310, 2001.

[4] H. Belgacem, T. Leduc, and M. Musy. Towards a QGIS-based Graph Carrier of Urban Information and Spotting Wind Behavior at the Pedestrian Level. In 10th International Conference on Urban Climate/14th Symposium on the Urban Environment. New York, USA, Aug. 2018.

[5] J. Bouyer, M. Musy, Y. Huang, and K. Athamena. Mitigating urban heat island effect by urban design: Forms and materials. Cities and Climate Change: Responding to an Urgent Agenda, pp. 164-181, 2011.

[6] A. Dasgupta, J. Poco, B. Rogowitz, K. Han, E. Bertini, and C. T. Silva. The effect of color scales on climate scientists' objective and subjective performance in spatial data analysis tasks. IEEE transactions on visualization and computer graphics, PP:1-1, 2018.

[7] V. Equere, P. A. Mirzaei, and S. Riffat. Definition of a new morphological parameter to improve prediction of urban heat island. Sustainable Cities and Society, 56:102-021, 2020.

[8] E. Hawkins. Warming stripes for 1850-2018 using the wmo annual global temperature dataset. Climate Lab Book. http://www.climate-labbook.ac.uk/2018/2018-visualisation-update/, 2018.

[9] G. Kristóf and B. Papp. Application of gpu-based large eddy simulation in urban dispersion studies. Atmosphere, 9(11):442, 2018.

[10] M. Kurppa, S. Karttunen, A. Hellsten, and L. Järvi. Including aerosol dynamic processes in LES: evaluation and application. In 100th American Meteorological Society Annual Meeting. Boston, USA, 2020.

[11] C. Lac, J.-P. Chaboureau, V. Masson, J.-P. Pinty, P. Tulet, J. Escobar, M. Leriche, C. Barthe, B. Aouizerats, C. Augros, et al. Overview of the meso-nh model version 5.4 and its applications. Geoscientific Model Development, 11(5):1929-1969, 2018.

[12] B. Le Roy, A. Lemonsu, R. Kounkou-Arnaud, D. Brion, and V. Masson. Long time series spatialized data for urban climatological studies: A case study of Paris, France. International Journal of Climatology, 40(7):3567-3584, 2020. doi: 10.1002/joc.6414

[13] M. J. Lobo, A. Telea, and C. Hurter. Feature Driven Combination of Animated Vector Field Visualizations. Computer Graphics Forum, 2020. doi: $10.1111 / \mathrm{cgf} .13992$

[14] V. Masson. A physically-based scheme for the urban energy budget in atmospheric models. Boundary-layer meteorology, 94(3):357-397, 2000.

[15] V. Masson, C. Marchadier, L. Adolphe, R. Aguejdad, P. Avner, M. Bonhomme, G. Bretagne, X. Briottet, B. Bueno, C. De Munck, et al. Adapting cities to climate change: A systemic modelling approach Urban Climate, 10:407-429, 2014.

[16] K. Moreland, C. Sewell, W. Usher, L. Lo, J. Meredith, D. Pugmire, J. Kress, H. Schroots, K. Ma, H. Childs, M. Larsen, C. Chen, R. Maynard, and B. Geveci. Vtk-m: Accelerating the visualization toolkit for massively threaded architectures. IEEE Computer Graphics and Applications, 36(3):48-58, 2016.

[17] T. R. Oke. Boundary layer climates. London: Routledge, 1987. doi: $10.4324 / 9780203407219$

[18] T. R. Oke, G. Mills, A. Christen, and J. A. Voogt. Urban Climates. Cambridge University Press, 2017. doi: 10.1017/9781139016476

[19] M. Parsaee, M. Joybari, P. A. Mirzaei, and F. Haghighat. Urban heat island, urban climate maps and urban development policies and action plans. Environmental technology \& innovation, pp. 100-341, 2019.

[20] L. Pinson and V. Masson. Heat stress in urban area: data fusion of observations, modeling and geospatial information. In First International Conference on Urban Physics (FICUP 2016). Sept, 2016. Quito, Ecuador, 2016.

[21] C. Plumejeaud-Perreau, C. Poitevin, C. Pignon-Mussaud, and N. Long. Building local climate zones by using socio-economic and topographic vectorial databases. In 9th International Conference on Urban Climate jointly with 12th Symposium on the Urban Environment. Jul, 2015. Toulouse, France., 2015.

[22] R. Ramyar, E. Zarghami, and M. Bryant. Spatio-temporal planning of urban neighborhoods in the context of global climate change: Lessons for urban form design in tehran, iran. Sustainable Cities and Society, $51: 101-554,2019$

[23] A. Rodler and T. Leduc. Local climate zone approach on local and micro scales: Dividing the urban open space. Urban Climate, 28:100457, 2019.

[24] M. Santamouris. Heat-island effect. Energy and climate in the urban built environment, p. 402, 2001.

[25] R. Schoetter, J. Hidalgo, R. Jougla, V. Masson, M. Goret, M. Rega, and J. Pergaud. A statistical-dynamical downscaling for the urban heat island and building energy consumption: Analysis of its uncertainties. Journal of Applied Meteorology and Climatology, 59:859-883, 2020.

[26] R. Stauffer, G. J. Mayr, M. Dabernig, and A. Zeileis. Somewhere over the rainbow: How to make effective use of colors in meteorological visualizations. Bulletin of the American Meteorological Society, 96(2):203-216, 2015.

[27] I. Stewart and T. Oke. Classifying urban climate field sites by "local climate zones": The case of nagano, japan. In 7th International Conference on Urban Climate (ICUC-7), vol. (29). Jun-Jul, 2009. Yokohama, Japan., 2009.

[28] I. D. Stewart and T. R. Oke. Local climate zones for urban temperature studies. Bulletin of the American Meteorological Society, 93(12):1879-1900, 2012

[29] N. Yaghoobian and J. Kleissl. An indoor-outdoor building energy simulator to study urban modification effects on building energy usemodel description and validation. Energy and Buildings, 54:407-417, 2012. 